\title{
Neues zum beruflichen Umgang mit Isocyanaten
}

\author{
New Data on Occupational Exposure to Isocyanates
}

Autoren

Institut
X. Baur, L. T. Budnik

Ordinariat und Zentralinstitut für Arbeitsmedizin und Maritime Medizin, Hamburg eingereicht 4.8.2009

akzeptiert nach Revision 10. 8. 2009

\section{Bibliografie}

DOI http://dx.doi.org/ 10.1055/s-0029-1215098

Online-Publikation: 18. 9. 2009

Pneumologie 2009; 63: 656-661 @ Georg Thieme Verlag KG Stuttgart · New York ISSN 0934-8387

\section{Korrespondenzadresse}

Prof. Dr. med. Xaver Baur Ordinariat und Zentralinstitut für Arbeitsmedizin und Maritime Medizin Seewartenstraße 10 20459 Hamburg baur@uke.uni-hamburg.de

\section{Zusammenfassung \\ $\nabla$}

In den letzten Jahrzehnten hat die industrielle Nutzung von Isocyanaten dank einer riesigen Produktpalette mit unterschiedlichsten Materialeigenschaften erheblich zugenommen. Dieser rasanten Entwicklung hinken diesbezüglich relevante Arbeitsschutzregularien weit hinterher. Gesundheitsschädigende Wirkungen gehen vor allem von der inhalativen Exposition gegenüber Isocyanaten aus. Daneben kann aber auch Hautkontakt zu toxischen und allergischen Reaktionen führen. Die heute technisch ganz im Vordergrund stehenden oligo- und polymeren Isocyanate werden lediglich in den Technischen Regeln für Gefahrstoffe (TRGS) 430, nicht aber in der rechtsverbindlichen Arbeitsmedizinischen Vorsorgeverordnung berücksichtigt. Trotz der z.T. bedenklichen Vereinfachungen der Risikoabschätzung (Berücksichtigung eines sich auf die Tröpfchengröße beziehenden Aerosol-Penetrationsfaktors; Beurteilung nur der tierexperimentell ermittelten akuten Reizwirkung der oligo- und polymeren Isocyanate bei der Festlegung des Beurteilungswertes) greift die TRGS 430 nach eigener Beobachtung bisher nicht in der Praxis.

\section{Einleitung}

\section{$\nabla$}

Isocyanate zählen weltweit zu den häufigsten Ursachen des berufsbedingten Asthma bronchiale und anderer Atemwegserkrankungen [1-4]. In Deutschland werden unter der Berufskrankheit Nummer 1315, die bis auf Hautaffektionen alle Isocyanat-bedingten Berufskrankheiten zusammenfasst, jährlich etwa 50 Fälle neu anerkannt. Die Diagnostik ist oft schwierig, da in lediglich etwa $20 \%$ der Fälle spezifische IgE-Antikörper nachweisbar sind und Arbeitsplatzsimulationsteste aufgrund ihres großen Aufwands nur in wenigen Zentren erfolgen [5]. Die geringe Sensitivität allergologischer Verfahren kann nur z.T. durch

\section{Abstract \\ $\nabla$}

During recent decades the industrial use of isocyanates has expanded enormously due to the huge product palette with a variety of different properties. As opposed to the fast technical development, there is a delay in the formulation of respective occupational health and safety regulations. Adverse health effects of isocyanates are mainly due to inhalative exposure. In addition, cutaneous contact causes toxic as well as allergic reactions. Oligomeric and polymeric isocyanates that are mostly used in the industry are only regulated in the Technical Rules for Hazardous Substances 430 (TRGS 430 ), but not by the mandatory occupational medical surveillance directive (Arbeitsmedizinische Vorsorgeverordnung). Although the TRGS 430 facilitates risk assessment (e.g., the aerosol penetration factor is related to the size of aerosols; only the acute irritative effect of oligomeric and polymeric isocyanates as shown in animal studies is taken into consideration in the case of defining a so-called evaluation factor), so far this new regulation has not yet been implemented in practice according to our own findings.

deren unzureichende Sensitivität mit nicht optimalen Isocyanat-Konjugaten erklärt werden. Es ist davon auszugehen, dass nicht-allergische Pathomechanismen dominieren.

\section{Arbeitsbedingte Expositionen \\ $\nabla$}

Isocyanate sind moderne Syntheseausgangsstoffe für die Herstellung von Weich-, Hart-, Integral-, Isolier-Schaumstoffen und anderen Kunststoffen, Lacken und sonstigen Oberflächen-Beschichtungen, Vergussmassen, Elastomeren, Klebern, Härtern, Pharmazeutika, Pestiziden und weiteren Erzeugnissen der chemischen Industrie. Anwen- 


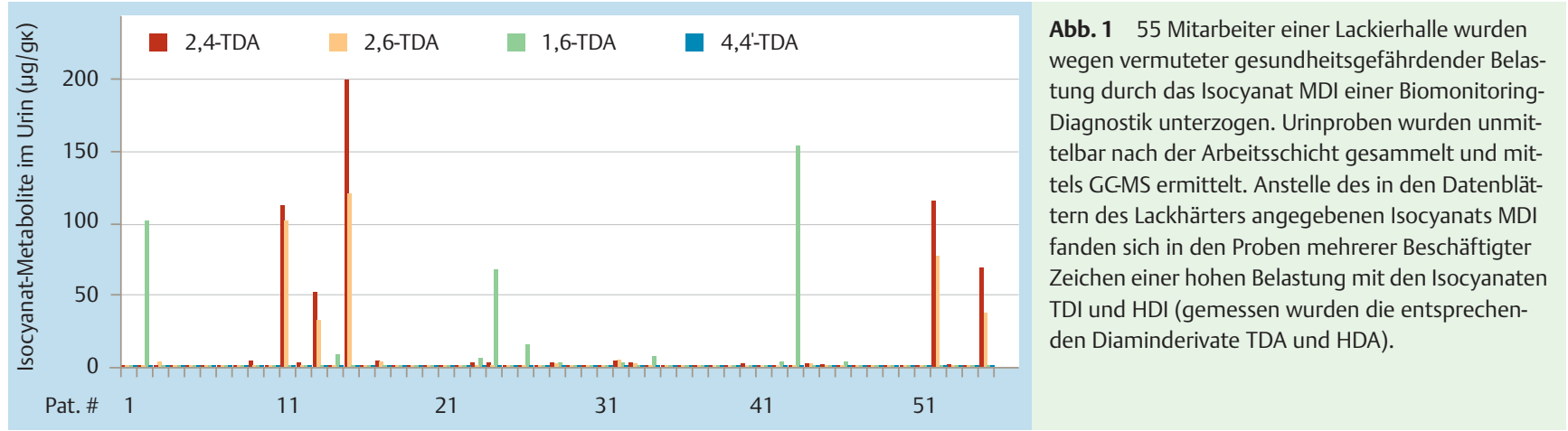

dungsbereiche sind vor allem die Kraftfahrzeug-, Flugzeug-, Metall-, Möbel-, Textil-, Bekleidungs- und holzverarbeitende Industrie, das Baugewerbe, der Bergbau (Gebirgsverfestigung), Gießereien und der Sportbahnbau. Gelegentlich werden Isocyanate mit anderen Systemen kombiniert, z. B. mit Epoxid-haltigen und Alkydharz-Bindemitteln.

Von gesundheitsrelevanter Bedeutung sind in erster Linie Isocyanat-haltige Dämpfe und Aerosole. Letztere entstehen vornehmlich beim Spritzlackieren von Lacken mit Isocyanathärtern. Da der Dampfdruck stark temperaturabhängig ist, können auch im Rahmen der exotherm ablaufenden Polyurethan(PUR)-Schaumstoffherstellung hohe Luftkonzentrationen auftreten. Mit einer Gesundheitsgefährdung muss sowohl bei der Anwendung von Isocyanat-haltigen 2-Komponenten-Reaktionssystemen (Reaktionspartner sind hier meist mehrwertige Alkohole, z. T. auch Amine und Wasser) als auch von 1-Komponenten-Produkten (diese härten mit dem Wasserdampf der Luft aus) gerechnet werden. Großflächig aufgetragen können Isocyanate durch verdunstende Lösemittel mitgerissen werden.

Beim Erhitzen, Verschwelen und Verbrennen von ausgehärteten Polyurethanen (PUR) werden neben anderen, großteils toxisch wirkenden Produkten verschiedene Isocyanate gebildet und freigesetzt. Dies gilt v.a. für das Schweißen von PUR-lackierten oder -beschichteten Metallen, für das Ein- oder Abbrennen von PURLackschichten, das Stahl- und Aluminiumgießen in MDI-gefestigten Sandkernen und anderen Formen, das Schneiden von Hartschaumplatten, die mechanische Bearbeitung unter Hitzeentwicklung von Isocyanat-verleimten Spanplatten, das Anschleifen von PUR-Anstrichen, Wohnungs- und Autobrände. Auch starkes Erhitzen und Verbrennen von stickstoffhaltigen Materialien, wie Phenol-Formaldehyd-Harnstoff-Harz (Bakelite, u. a. Chip-Platinen) und beschichteter Steinwolle, können zur Bildung und Freisetzung von Isocyanaten führen [6-8]. In der Regel entstehen bei den vorgenannten Prozessen unter hohen Temperaturen (über $350^{\circ} \mathrm{C}$ ) niedermolekulare Monoisocyanatverbindungen wie Isocyansäure (HNCO; ICA) und Methylisocyanat $\left(\mathrm{CH}_{2} \mathrm{NCO}\right.$; $\left.\mathrm{MIC}\right)$.

Neben der inhalativen Aufnahme kann Hautkontakt zu einer Isocyanat-Inkorporation führen. Hierdurch werden nach tierexperimentellen Befunden sowohl lokale Reizerscheinungen als auch respiratorische Überempfindlichkeitsreaktionen ausgelöst [910].

Peroral ist eine Isocyanat-Aufnahme beim Rauchen oder beim Essen im Arbeitsbereich möglich.

\section{Was wissen wir über die heutigen Arbeitsplatzkonzentrationen? \\ $\nabla$}

In der heutigen Arbeitswelt werden ganz überwiegend oligound polymere Isocyanate eingesetzt. Ihre Luftkonzentration hängt von den jeweiligen Arbeitsbedingungen, der Temperatur und den verarbeiteten Mengen ab. Woskie et al. [11] wiesen NCO-Konzentrationen zwischen 0,02 und $3120 \mu \mathrm{g} / \mathrm{m}^{3}$ Luft in Lackierereien nach. Ähnliche Ergebnisse fanden Pronk et al. [12]. Auch Untersuchungen in England ergaben in Lackierereien und in der Elastomeren-Herstellung teilweise erhöhte Isocyanat-Luftkonzentrationen [13-14] s. auch $\bullet$ Abb. 1. Entsprechende Aussagen lieferten neuere Biomonitoringuntersuchungen bei verschiedenen Isocyanatanwendungen [13 - 20]. Dabei konnte Jones [21] belegen, dass die Aufklärung der Beschäftigten zu einer beachtlichen Verbesserung der Arbeitshygiene und zur Reduktion der inneren Belastung führte.

\section{Arbeitsplatzregularien \\ $\nabla$}

Einen Biologischen Grenzwert (BGW; TRGS 903) [22] gibt es lediglich für das Diisocyanat MDI $(10 \mu \mathrm{g} / \mathrm{g}$ Kreatinin für den im Urin nachweisbaren Metaboliten 4,4'-Diaminodiphenylmethan, MDA).

Anlage 1 gibt die aktuellen rechtsverbindlichen Arbeitsplatzgrenzwerte (AGW) der Isocyanate wieder, die sich ausschließlich auf Di- und zwei Monoisocyanate beziehen und im Allgemeinen bei 5 ppb liegen. Für die industriell ganz im Vordergrund stehenden oligo- und polymeren Isocyanate wurden in Deutschland bisher keine Arbeitsplatzgrenzwerte festgesetzt.

Die American Conference of Governmental Industrial Hygienists [23] schlug kürzlich für TDI einen deutlich niedrigeren Arbeitsplatzgrenzwert (TWA, 8-Stunden-Mittelwert), nämlich von $0,007 \mathrm{mg} / \mathrm{m}^{3}$ (1 ppb), und einen 15-Minuten-Kurzzeitwert von $0,021 \mathrm{mg} / \mathrm{m}^{3}$ (3 ppb) vor.

Die Technischen Regeln für Gefahrstoffe (TRGS) 430 „Isocyanate Exposition und Überwachung“ [24], aktualisiert im März 2009, beinhalten eine umfassende Darstellung der Isocyanatanwendungen in den verschiedensten Industriezweigen. Diese ist hilfreich für die vom Arbeitgeber zu erstellende Gefährdungsbeurteilung, die die Gesamt-Isocyanatexposition anhand eines Bewertungsindex berücksichtigen soll, also auch die in Aerosolen befindlichen Isocyanatanteile. Dabei werden ein sog. Aerosol-Penetrationsfaktor (basiert auf dem Durchmesser der Aerosole) und bei oligomeren und polymeren Isocyanaten ein Expositionsbeurteilungswert (EBW; erfasst ausschließlich die lokale Reizwirkung und ist vom Hersteller anzugeben) zugrunde gelegt. Die Intensi- 
tät der Exposition wird im Einzelnen unter Berücksichtigung folgender Parameter beurteilt:

- der klinischen Natur des Isocyanates,

- dem Arbeitsplatzgrenzwert für Diisocyanate nach TRGS 900 [25] „Luftgrenzwerte in der Luft am Arbeitsplatz“ und Arbeitsmedizinischer Vorsorgeverordnung (2009),

- der Dampfbildung (durch Aerosole aus dem Kondensat),

- der Aerosolbildung (durch Aerosole aus dem Applikationsverfahren); bei Oligo- und Polymeren s. Beurteilungswert,

- dem Eindringvermögen in die Lunge (Aerosol-Penetrationsfaktor, APF),

- dem Ausmaß des Hautkontaktes.

Der Beurteilungsindex (BI) für den jeweiligen Arbeitsplatz leitet sich aus der Summe der Verhältniszahlen a) der Konzentration (C) der monomeren Isocyanate (i)/AGW und b) der Konzentration der oligo- und polymeren Isocyanate (Poly) $\times$ APF/EBW ab:

$\mathrm{BI}=\Sigma\left(\mathrm{C}_{\mathrm{i}} / \mathrm{AGW}_{\mathrm{i}}+\mathrm{C}_{\text {Poly }} \times \mathrm{APF} / \mathrm{EBW}\right)$

Neu ist die in der TRGS 430 [24] vorgenommene Festlegung eines Expositionsleitwertes, der alle Isocyanate in der Raumluft berücksichtigt und $0,018 \mathrm{mg} / \mathrm{m}^{3}$ beträgt.

\section{Arbeitsmedizinische Vorsorgeuntersuchung [26]}

Isocyanat-exponierte Beschäftigte sind einer speziellen arbeitsmedizinischen Vorsorgeuntersuchung [27] zu unterziehen, wenn die Luftkonzentration $0,05 \mu \mathrm{g} / \mathrm{m}^{3}$ überschreitet oder wenn regelmäßiger oder ständiger Hautkontakt gegeben ist [28]. Unverständlicherweise wurde in dieser neuen rechtsverbindlichen Verordnung die bisherige Verpflichtung des Arbeitgebers zu einer Angebotsuntersuchung bei geringerer Exposition nicht übernommen.

Zu Recht wird in der Arbeitsmedizinischen Vorsorge G 27 [27] auf das erhöhte Gesundheitsrisiko von Personen mit vorbestehenden Atemwegserkrankungen und bronchialer Hyperreagibilität hingewiesen. Nicht richtig ist allerdings, dass Personen mit umweltbedingten Allergien ein erhöhtes Gesundheitsrisiko haben. Das Gegenteil ist der Fall [29].

\section{Notwendigkeit einer verbesserten Prävention}

Eine Vielzahl von Untersuchungen belegt, dass die gesundheitsadversen Effekte der Isocyanate konzentrationsabhängig sind $[4,30,31]$. Durch geeignete primärpräventive Maßnahmen (geschlossene Systeme, verbesserte Luftabsaugungen, elektrostatisch arbeitende Spritzverfahren etc.) und durch Sekundärprävention (Atemmasken, Schutzanzüge, Handschuhe) lässt sich die Exposition in praktisch allen Arbeitsbereichen in gesundheitlich unbedenkliche Bereiche absenken.

Die in Deutschland nach wie vor ausschließlich für Di- und Monoisocyanate geltenden Arbeitsplatzgrenzwerte erfuhren, wie dargestellt, nun eine Ergänzung in der TRGS 430 mit Berücksichtigung der Gesamt-Isocyanat-Belastung. Allerdings geht in den dort aufgeführten, eher einem sicherheitstechnischen Standard entsprechenden Bewertungsindex nur die lokale, tierexperimentell ermittelte Atemwegsirritation ein, anzunehmende verzögerte Reizwirkungen, Allergenität und evtl. Kanzerogenität bleiben hierbei unberücksichtigt. Es bleibt zu hoffen, dass der Arbeitsschutz beim Umgang mit Isocyanaten (Probleme bestehen vorwiegend in Klein- und Mittelbetrieben) durch Umsetzung dieser TRGS und durch Schaffung einer besseren Datenbasis bezüglich der nach wie vor nicht befriedigenden Risikoabschätzung der oligo- und polymeren Isocyanate optimiert wird.

\section{Interessenkonflikte}

Die Autoren erklären, dass kein Interessenkonflikt besteht.

\section{Literatur}

1 Goe SK, Henneberger PK, Reilly MJ et al. A descriptive study of work aggravated asthma. Occup Environ Med 2004; 61: 512-517

2 Vandenplas O, Malo JL, Saetta $\mathrm{M}$ et al. Occupational asthma and extrinsic alveolitis due to isocyanates: current status and perspectives. $\mathrm{Br} J$ Ind Med 1993; 50: 213-228

3 Bernstein JA. Overview of diisocyanate occupational asthma. Toxycology 1996; 111: $181-189$

4 Baur X. Occupational asthma due to isocyanates. Lung 1996; 174: 23 30

5 Baur X, Latza U, Barbinova L, Yu F. Isocyanat-bedingte Gesundheitsgefahren - eine aktuelle Literaturübersicht. Arbeitsmed Sozialmed Umweltmed 2003; 38: 270

6 Karlsson D, Spanne M, Skarping G. Airborne thermal degradation products of polyurethane coatings in car repair shops. J Environ Monit 2000; 2: 462-469

7 Karlsson D, Dalene M, Skarping G. A Manrad Determination of isocyanaic acid in air. J Environ Monit 2001; 3: 432-436

8 Karlsson D, Dahlin J, Skarping G, Dalene M. Determination of isocyanates, aminoisocyanates and amines in air formed during thermal degradation of polyurethane. J Environ Monit 2002; 4: 216-222

9 Redlich CA, Herrick CA. Lung/skin connections in occupational lung disease. Curr Opin Allergy Clin Immunol 2008; 8: 115-119

10 Cocker J. Biological monitoring for isocyanates. Occup Med 2007; 57: $391-393$

11 Woskie SR, Sparer J, Gore RJ et al. Determinants of isocyanate exposures in auto body repair and refinishing shops. Ann Occup Hyg 2004; 48: $393-403$

12 Pronk A, Preller L, Raulf-Heimsoth $M$ et al. Respiratory symptoms, sensitization, and exposure response relationships in spray painters exposed to isocyanates. Am J Respir Crit Care Med 2007; 176: 1090 1097

13 Creely KS, Hughson GW, Cocker J, Jones K. Assessing isocyanate exposures in polyurethane industry sectors using biological and air monitoring methods. Ann Occup Hyg 2006; 50: 609-621

14 Cocker J, Cain JR, Baldwin P et al. A survey of occupational exposure to 4,4'-methylene-bis (2-chloroaniline) (MbOCA) in the UK. Ann Occup Hyg 2009; [Epub ahead of print]

15 Schutze D, Sepai 0 , Lewalter J et al. Biomonitoring of workers exposed to $4,4^{\prime}$-methylenedianiline or $4,4^{\prime}$-methylenediphenyl diisocyanate. Carcinogenesis 1995; 16: $573-582$

16 Kääriä K, Hirvonen A, Norppa $H$ et al. Exposure to 2,4- and 2,6-toluene diisocyanate (TDI) during production of flexible foam: determination of airborne TDI and urinary 2,4- and 2,6-toluenediamine (TDA). Analyst 2001; 126: $1025-1031$

17 Pronk A, Tielemans E, Skarping $G$ et al. Inhalation exposure to isocyanates of car body repair shop workers and industrial spray painters. Ann Occup Hyg 2006; 50: 1 - 14

18 Sabbioni G, Wesp H, Lewalter J et al. Determination of isocyanate biomarkers in construction site workers. Biomarkers 2007; 12: 468 - 483

19 Budnik LT et al. Effective biological monitoring for isocyanates - toxicological kinetics in patients after specific inhalation challenges. In Vorbereitung

20 Austin S. Biological monitoring of TDI-derived amines in polyurethane foam production. Occup Med 2007; 57: $444-448$

21 Jones $K$. Biological monitoring isocyanates: A guidance value and its applications. http://www.bohs.org/resources/res.aspx/Resource/filename/1011/Biological_Monitoring_for_Isocyanates_A_Guidance_Value_and_its_Application.pdf; Stand: 1.7.2009

22 TGRS 903 (Technische Regeln für Gefahrstoffe). Biologische Grenzwerte 2006: $1-7$

23 American Conference of Governmental Industrial Hygienists (ACGIH). 2009 TLVs® and BEIs®. Based on the Documentation of the Threshold Limit Values for chemical substances and physical agents \& biological exposure indices. Cincinnati, Ohio: ACGIH, 2009 
24 TRGS 430 (Technische Regeln für Gefahrstoffe). Isocyanate - Gefährdungsbeurteilung und Schutzmaßnahmen. GMBl 2009; 18/19: 349382

25 TRGS 900 (Technische Regeln für Gefahrstoffe). Arbeitsplatzgrenzwerte. GMBl 2009; 28: 605

26 Bundesgesetzblatt 2008. Verordnung zur Rechtsvereinfachung und Stärkung der arbeitsmedizinischen Vorsorge - Art. 1: Verordnung zur arbeitsmedizinischen Vorsorge (ArbMedVV) vom 18.12.2008 (BGBI. IS. 2768). Bundesgesetzblatt Teil I Nr. 62, ausgegeben zu Bonn am 23. $12.2008: 2768$
27 Deutsche Gesetzliche Unfallversicherung (DGUV). Berufsgenossenschaftliche Grundsätze für arbeitsmedizinische Vorsorgeuntersuchungen. 4. Auflage. Stuttgart: Gentner, 2007

28 Verordnung zur Rechtsvereinfachung und Stärkung der arbeitsmedizinischen Vorsorge - Art. 1: Verordnung zur arbeitsmedizinischen Vorsorge (ArbMedVV) vom 18. 12. 2008. BGBl I 2008: 2768-2776

29 Baur X, Barbinova L. Occupational exposures, specific sensitizations and the atopic status. Eingereicht

30 Redlich CA, Karol MH. Diisocyanate asthma: clinical aspects and immunopathogenesis. Int Immunnopharmacol 2002; 2: 213-224

31 Silk SJ, Hardy HL. Control limits for isocyanates. Ann Occup Hyg 1983; 27: $333-339$ 


\section{Anlage 1 zu TRGS 430}

\section{Einstufung, Kennzeichnung und Arbeitsplatzgrenzwerte einiger Isocyanate}

Stand der Information:

1. Einstufung: Verordnung (EG) Nr. $1272 / 2008$ des Europäischen Parlaments und des Rates vom 16. Dezember 2008 über die Einstufung, Kennzeichnung und Verpackung von Stoffen und Gemischen, Anhang VI, Tabelle 3.2 (http://eur-lex.europa.eu/JOHtml.do?uri=OJ:L:2008:353:SOM:DE:HTML)

2. Arbeitsplatzgrenzwerte: TRGS 900, Ausgabe: Januar 2006; zuletzt geändert und ergänzt: GMBI Nr. 12-14 S. 254 (27.3.2009)

\begin{tabular}{|c|c|c|c|c|c|}
\hline \multirow{2}{*}{$\begin{array}{l}\text { Stoffidentität } \\
\text { EG-Nr. } \\
\text { CAS-Nr. } \\
\end{array}$} & \multirow[t]{2}{*}{ Einstufung } & \multicolumn{4}{|c|}{ Arbeitsplatzgrenzwerte } \\
\hline & & $\mathrm{mg} / \mathrm{m}^{3}$ & $\begin{array}{l}\mathrm{ml} / \mathrm{m}^{3} \\
(\mathrm{ppm})\end{array}$ & $\begin{array}{l}\text { Spitzenbe- } \\
\text { grenzung }\end{array}$ & Bemerkungen \\
\hline \multicolumn{6}{|l|}{ Aromatische Diisocyanate } \\
\hline 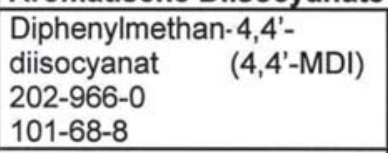 & $\begin{array}{c}\text { Carc. Cat. 3; R40* } \\
\text { Xn; R20-R48/20 } \\
\text { Xi; R36/37/38 } \\
\text { R42/43 } \\
\end{array}$ & 0,05 & & $1 ;=2=(I)$ & $11,12, \mathrm{Sa}$ \\
\hline $\begin{array}{ll}\text { Diphenylmethan-2,4'- } \\
\text { diisocyanat } \quad\left(2,4^{\prime}-\mathrm{MDI}\right) \\
227-534-9 & \\
5873-54-1 & \\
\end{array}$ & $\begin{array}{c}\text { Carc. Cat. } 3 ; R_{40}^{*} \\
\text { Xn; R20-R48/20 } \\
\text { Xi; R36/37/38 } \\
\text { R42/43 } \\
\end{array}$ & 0,05 & & $1 ;=2=(I)$ & $11,12, \mathrm{Sa}$ \\
\hline $\begin{array}{ll}\text { Diphenylmethan-2,2'- } \\
\text { diisocyanat } \quad\left(2,2^{\prime}-M D I\right) \\
219-799-4 & \\
2536-05-2 & \\
\end{array}$ & $\begin{array}{c}\text { Carc. Cat. 3; R40* } \\
\text { Xn; R20-R48/20 } \\
\text { Xi; R36/37/38 } \\
\text { R42/43 }\end{array}$ & 0,05 & & $1 ;=2=(I)$ & $11,12, \mathrm{Sa}$ \\
\hline \begin{tabular}{|ll} 
Diphenylmethan- & \\
diisocyanat, & \\
Isomerengemisch & (MDI) \\
$247-714-0$ & \\
$26447-40-5$ & \\
\end{tabular} & $\begin{array}{c}\text { Carc. Cat. 3; R40* } \\
\text { Xn; R20-R48/20 } \\
\text { Xi; R36/37/38 } \\
\text { R42/43 } \\
\end{array}$ & & & & $\begin{array}{l}\quad 11,12, \mathrm{Sa} \\
\text { Es gelten die AGW der } \\
\text { Einzelisomere }\end{array}$ \\
\hline $\begin{array}{l}\begin{array}{l}\text { Diphenylmethan- } \\
\text { diisocyanat, } \\
\text { Homologe }\end{array} \begin{array}{r}\text { (somere und } \\
\text { (p-MDI }\end{array} \\
\begin{array}{ll}\text { (techn. MDI) }\end{array} \\
9016-87-9\end{array}$ & $\begin{array}{c}\text { Hersteller- } \\
\text { einstufung } \\
\text { beachten } \\
\text { (einatembare Frak- } \\
\text { tion: Carc. Cat } 3^{*} \text { ) }\end{array}$ & & & & $\begin{array}{l}\text { Für den Zweikern-Anteil } \\
\text { gelten die AGW der } \\
\text { Einzelisomere (4,4'-MDI, } \\
\text { 2,4'-MDI, 2,2'-MDI); für } \\
\text { den Homologen-Anteil } \\
\text { ist der EBW (Hersteller- } \\
\text { angabe) heranzuziehen } \\
\end{array}$ \\
\hline \begin{tabular}{ll|} 
Naphthylen-1,5- & \\
diisocyanat & (NDI) \\
$221-641-4$ & \\
$3173-72-6$ & \\
\end{tabular} & $\begin{array}{c}\mathrm{Xn} ; \mathrm{R} 20 \\
\mathrm{Xi} ; \mathrm{R} 36 / 37 / 38 \\
\mathrm{R} 42 \\
\mathrm{R} 52-53 \\
\end{array}$ & 0,05 & & $1 ;=2=(I)$ & AGS, $11,12, \mathrm{Sa}$ \\
\hline $\begin{array}{l}\text { 2,4-Diisocyanattoluol } \\
\begin{array}{l}\text { (2,4-TDI) } \\
209-544-5 \quad \\
584-84-9\end{array}\end{array}$ & $\begin{array}{c}\text { Carc. Cat. 3; R40 } \\
\text { T+; R26 } \\
\text { Xi; R36/37/38 } \\
\text { R42/43 } \\
\text { R52-53 }\end{array}$ & 0,035 & 0,005 & $1 ;=4=(I)$ & AGS, 12, Sa \\
\hline $\begin{array}{l}\text { 2,6-Diisocyanattoluol } \\
\begin{array}{l}\text { (2,6-TDI) } \\
202-039-0 \quad \\
91-08-7\end{array} \\
\end{array}$ & $\begin{array}{c}\text { Carc. Cat. 3; R40 } \\
\text { T+; R26 } \\
\text { Xi; R36/37/38 } \\
\text { R42/43 } \\
\text { R52-53 } \\
\end{array}$ & 0,035 & 0,005 & $1 ;=4=(I)$ & AGS, 12, Sa \\
\hline $\begin{array}{lr}\text { m-Diisocyanattoluol (1,3-) } & (2,4-/ 2,6-\text { TDI) } \\
& (m-T D I) \\
247-722-4 & \\
26471-62-5 & \\
\end{array}$ & $\begin{array}{c}\text { Carc. Cat. 3; R40 } \\
\text { T+; R26 } \\
\text { Xi; R36/37/38 } \\
\text { R42/43 } \\
\text { R52-53 } \\
\end{array}$ & & & & $\begin{array}{l}\text { Für das 2,4-/2,6-TDI } \\
\text { Isomerengemisch sind } \\
\text { die AGW der Einzel- } \\
\text { isomere 2,4-TDI bzw. } \\
\text { 2,6-TDI heranzuziehen } \\
\end{array}$ \\
\hline \multicolumn{6}{|l|}{ Aliphatische Diisocyan } \\
\hline $\begin{array}{ll}\text { Hexamethylen-1,6- } & \\
\text { diisocyanat } & \text { (HDI) } \\
212-485-8 & \\
822-06-0 & \\
\end{array}$ & $\begin{aligned} & \mathrm{T} ; \mathrm{R} 23 \\
& \mathrm{Xi} ; \mathrm{R} 36 / 37 / 38 \\
& \mathrm{R} 42 / 43\end{aligned}$ & 0,035 & 0,005 & $1 ;=2=(I)$ & DFG, $12, \mathrm{Sa}$ \\
\hline $\begin{array}{l}2,2,4-\text { Trimethylhexa- } \\
\text { methylen-1,6-diisocyanat }\end{array}$ & $\begin{array}{c}\mathrm{T} ; \mathrm{R} 23 \\
\mathrm{Xi} ; \mathrm{R} 36 / 37 / 38 \\
\end{array}$ & & & & $\begin{array}{c}\text { Herstellerempfehlung } \\
\text { beachten }\end{array}$ \\
\hline
\end{tabular}




\begin{tabular}{|c|c|c|c|c|c|}
\hline \multirow{2}{*}{$\begin{array}{l}\text { Stoffidentität } \\
\text { EG-Nr. } \\
\text { CAS-Nr. }\end{array}$} & \multirow{2}{*}{ Einstufung } & \multicolumn{4}{|c|}{ Arbeitsplatzgrenzwerte } \\
\hline & & $\mathrm{mg} / \mathrm{m}^{3}$ & $\begin{array}{l}\mathrm{ml} / \mathrm{m}^{3} \\
(\mathrm{ppm})\end{array}$ & $\begin{array}{l}\text { Spitzenbe- } \\
\text { grenzung }\end{array}$ & Bemerkungen \\
\hline $\begin{array}{ll}241-001-8 & \text { (TMDI) } \\
16938-22-0 & \\
\end{array}$ & R42 & & & & \\
\hline $\begin{array}{l}\text { 2,4,4-Trimethylhexa- } \\
\text { methylen-1,6-diisocyanat } \\
239-714-4 \quad \text { (TMDI) } \\
15646-96-5\end{array}$ & $\begin{array}{c}\mathrm{T} ; \mathrm{R} 23 \\
\mathrm{Xi} ; \mathrm{R} 36 / 37 / 38 \\
\mathrm{R} 42 \\
\end{array}$ & & & & $\begin{array}{l}\text { Herstellerempfehlung } \\
\text { beachten }\end{array}$ \\
\hline $\begin{array}{l}1,3-\text { Tetramethylxylylen- } \\
\text { diisocyanat } \\
220-474-4 \\
2778-42-9\end{array}$ & $\begin{array}{l}\text { Hersteller- } \\
\text { einstufung } \\
\text { beachten }\end{array}$ & & & & $\begin{array}{l}\text { Herstellerempfehlung } \\
\text { beachten }\end{array}$ \\
\hline $\begin{array}{lr}1,4-\text { Tetramethylxylylen- } \\
\text { diisocyanat } & \text { (p-TMXDI) } \\
220-473-9 & \\
2778-41-8 & \\
\end{array}$ & $\begin{array}{l}\text { Hersteller- } \\
\text { einstufung } \\
\text { beachten }\end{array}$ & & & & $\begin{array}{l}\text { Herstellerempfehlung } \\
\text { beachten }\end{array}$ \\
\hline \multicolumn{6}{|c|}{ Cycloaliphatische Diisocyanate } \\
\hline $\begin{array}{l}\text { 3-Isocyanatmethyl-3,5,5- } \\
\text { trimethylcyclohexyl- } \\
\text { isocyanat } \\
\text { (Isophorondiisocyanat) } \\
223-861-6 \\
4098-71-9\end{array}$ & $\begin{array}{c}\text { T: R23 } \\
\text { Xi; } R 36 / 37 / 38 \\
\text { R42/43 } \\
\text { N; R51-53 }\end{array}$ & 0,046 & 0,005 & $1 ;=2=(I)$ & DFG, $12, \mathrm{Sa}$ \\
\hline $\begin{array}{lr}\text { Dicyclohexylmethan-4,4'- } \\
\begin{array}{l}\text { diisocyanat } \\
225-863-2\end{array} \\
5124-30-1 & \left.\text { ( } \text { ( }_{12} \text { MDI }\right) \\
\end{array}$ & $\begin{aligned} \mathrm{T} ; \mathrm{R} 23 \\
\mathrm{Xi} ; \mathrm{R} 36 / 37 / 38 \\
\mathrm{R} 42 / 43\end{aligned}$ & 0,054 & & & $\begin{array}{c}\text { (Bearbeitungsliste zur } \\
\text { TRGS 900) } \\
\text { NL, H, 29, } 36\end{array}$ \\
\hline $\begin{array}{l}2,5 \text {-(und 2,6-)Bis(isocya } \\
\text { natomethyl)-bicyclo[2.2.1] } \\
\text { heptan (Norbornandiisocy- } \\
\text { anat) } \\
411-280-2 \\
- \\
\end{array}$ & $\begin{array}{l}\text { T+; R26 } \\
\text { Xn; R22 } \\
\text { C; R34 } \\
\text { R42/43 } \\
\text { R52-53 }\end{array}$ & 0,045 & 0,005 & & AGS \\
\hline \multicolumn{6}{|l|}{ Monoisocyanate } \\
\hline $\begin{array}{l}\text { Methylisocyanat } \quad \text { MIC } \\
210-866-3 \\
624-83-9\end{array}$ & $\begin{array}{c}\text { F+; R12 } \\
\text { Repr. Cat. 3; R63 } \\
\text { T+; R26 } \\
\text { T; R24/25 } \\
\text { R42/43 } \\
\text { Xi; R37/38-41 } \\
\end{array}$ & 0,024 & 0,01 & $1(\mathrm{I})$ & DFG, H, 12 \\
\hline $\begin{array}{ll}\text { Phenylisocyanat } & \text { Phl } \\
203-137-6 & \\
103-71-9 & \\
\end{array}$ & $\begin{array}{l}\text { Hersteller- } \\
\text { einstufung } \\
\text { beachten }\end{array}$ & 0,05 & 0,01 & 1(I) & AGS, $12, \mathrm{Sa}$ \\
\hline $\begin{array}{l}\text { 4-Toluensulfonylisocyanat } \\
223-810-8 \\
4083-64-1\end{array}$ & $\begin{array}{c}\mathrm{R} 14 \\
\mathrm{Xi} ; \mathrm{R} 36 / 37 / 38 \\
\mathrm{R} 42\end{array}$ & & & & $\begin{array}{l}\text { Herstellerempfehlung } \\
\text { beachten }\end{array}$ \\
\hline
\end{tabular}

Bemerkungen TRGS 900: 11 (29): Summe aus Dampf und Aerosolen.

12 (36): Der Arbeitsplatzgrenzwert gilt in der Regel nur für die Monomeren.

Zur Beurteilung von Oligomeren oder Poymeren siehe TRGS 430 "Isocyanate".

$\mathrm{H}$ : (hautresorptiv),

Sa: atemwegssensibilisierende Stoffe.

* Einstufung gemäß 1. Ånderung der Verordnung (EG) Nr. 1272/2008 (in Vorbereitung) 